Relations industrielles

Industrial Relations

\title{
B.I.T. : Technological Change : The Tripartite Response, 1982-85. Genève, Bureau international du Travail, 1985, 355 pp., ISBN 92-2-105162-5
}

\section{Rolland Hurtubise}

\section{Volume 41, numéro 2, 1986}

URI : https://id.erudit.org/iderudit/050221ar

DOI : https://doi.org/10.7202/050221ar

Aller au sommaire du numéro

Éditeur(s)

Département des relations industrielles de l'Université Laval

ISSN

0034-379X (imprimé)

1703-8138 (numérique)

Découvrir la revue

Citer ce compte rendu

Hurtubise, R. (1986). Compte rendu de [B.I.T. : Technological Change : The Tripartite Response, 1982-85. Genève, Bureau international du Travail, 1985, 355 pp., ISBN 92-2-105162-5]. Relations industrielles / Industrial Relations, 41(2), 433-435. https://doi.org/10.7202/050221ar

Tous droits réservés (C) Département des relations industrielles de l'Université Laval, 1986
Ce document est protégé par la loi sur le droit d'auteur. L’utilisation des services d’Érudit (y compris la reproduction) est assujettie à sa politique d'utilisation que vous pouvez consulter en ligne.

https://apropos.erudit.org/fr/usagers/politique-dutilisation/ 
and unions in matters, at least, of the work environment which is directly linked to worker health and safety - a tantamount value. While QWL does produce «partial» benefits through an improved «human relations" style of management, it becomes evident that you cannot have full «humanization» without democratization. Only through a genuine democratization of industry will the primary work group have its own interest, not necessarily coincidental with the goals of shareholders and managers. In this regard, workers ought to meet with managers and their professional agents as free and equals in an industrial partnership. This organization of industry would have enormous benefits for society in terms of productivity and efficiency and more importantly for worker health, safety, and moral and spiritual development. Soft Sell: «Quality of Working Life» Programs and the Productivity Race by Don Wells contributes enormously toward this end. The author, I am certain, will be in the gratitude of Canadian workers for his inciteful analysis and expose of a growing management development. I recommend «Soft Sell» as required reading for workers, trade unionists, and students of industrial relations and management studies.

Robert SASS

University of Saskatchewan

Technological Change: The Tripartite Response, 1982-85, par Bureau international du travail, Genève, 1985, 355 pp. ISBN 92-2-105162-5

Cet analyse est composé d'articles parus dans la revue Social and Labour Bulletin du Bureau international du travail (B.I.T.). Il a pour objet de compléter et de mettre à jour les renseignements contenus dans New Technologies: Their Impact on Employment and the Working Environment qui fut publié par le B.I.T. en 1982.

Il illustre de façon plutôt «télégraphique» comment les secteurs public (les gouvernements) et privé (les industries) font face à «l'explosion» de la technologie nouvelle.

Plusieurs sujets sont examinés: les conventions collectives, le phénomène du travail à domicile, la vie privée, le transfert outre-frontières des données, etc. Aussi, une attention particulière est accordée à un secteur qui en préoccupe plusieurs: l'impact de la technologie sur les pays en voie de développement et d'industrialisation.

Il se dégage un thème central voulant qu'une relation nouvelle s'établisse entre les travailleurs et les nouveaux "outils» technologiques dont ils devront se servir. Bref, le lecteur découvre un ensemble d'études, relié à l'avenir de l'emploi, qui permet de dégager des éléments essentiels devant être considérés lors des ententes employeurs-employés.

Le point fort et fascinant de ce livre est qu'il offre des perspectives nationales et internationales.

Tel que mentionné, il s'agit d'un ensemble d'articles du Social and Labour Bulletin (SLB) qui tente de démontrer comment les problèmes reliés à la technologie nouvelle ont évolué, ont été étudiés et quelles solutions ont été proposées en fonction des différents contextes nationaux. Les deux côtés de la «médaille» technologique sont présentés: d'une part, les technologies nouvelles peuvent améliorer la qualité de vie; d'autre part, elles peuvent aussi occasionner le chômage et élargir l'écart entre les pays industrialisés et les pays en voie de développement. 


\section{Ce livre comprend dix chapitres:}

- un premier chapitre qui offre les réactions d'universitaires, de travailleurs et d'employeurs face au changement technologique. On y présente diverses solutions sous forme de stratégies qui assureraient une mise en oeuvre sécuritaire et équitable de la technologie nouvelle;

- les chapitres 2 et 3 examinent l'impact du changement technologique sur des secteurs industriels spécifiques, nommément, l'industrie de l'automobile et les télécommunications. Les répercussions sociales se traduisant en termes de création et de perte d'emplois sont également étudiées;

- le quatrième chapitre fait état de quelques-uns des problèmes du transfert technologique vers les pays en voie de développement et présente divers points de vue sur la technologie appropriée pour ces pays. Le lecteur formulera son opinion à savoir si l'emploi des ordinateurs personnels dans les pays du Tiers-Monde constitue un outil de développement approprié;

- le chapitre 5 discute des relations industrielles et comment la technologie nouvelle crée des attitudes nouvelles, ainsi que des patterns de négociations. Notamment, dans certains pays, la législation et les décisions des tribunaux du travail permettent à la main-d'oeuvre d'être impliquée de très près à la prise de décisions en matière de technologie;

- le sixième chapitre comprend un éventail représentatif des principales ententes survenues aux niveaux central et sectoriel, ainsi qu'au niveau des usines dans les pays industrialisés. On y retrouve des lignes directrices émergeant des organisations syndicales et patronales;

- le chapitre 7 cerne les préoccupations reliées à la vie privée et à la confidentialité des données et illustre les problèmes posés par les flux «trans-frontières» de données;

- le huitième chapitre offre des exemples de l'impact direct de la technologie sur la qualité et la quantité des emplois dans des firmes et secteurs et révèle un grand nombre de scénarios de conflits possibles. Il ressort de ce chapitre un message clé: lorsqu'on s'adapte rapidement aux besoins d'habilités changeantes imposées par les technologies, il est probable que de nouveaux emplois seront créés;

- l'avant dernier chapitre discute de sujets plus directement reliés à la bureautique et traite de façon détaillée des écrans de visualisation (les écrans cathodiques) et des sujets connexes dont, la classification et la sécurité d'emploi, les heures de travail, la sécurité et la santé au travail, etc.;

- finalement, le dixième et dernier chapitre examine les réactions de neuf pays a la bureautique. Des prévisions sur le plan international sont aussi émises.

Le livre contient une courte bibliographie et un index.

Le cas (ou les cas!) du Canada est discuté à très peu d'endroit dans ce livre. Par exemple, au premier chapitre traitant de l'informatisation et des sujets de politiques, on ne fait qu'effleurer les travaux du groupe de travail gouvernemental sur la micro-électronique et l'emploi qui fut créé en mars, 1982. On mentionne une de leurs conclusions: les efforts canadiens en matière de mise en oeuvre de la haute technologie sont «sans gouvernail»; il n'existe aucune stratégie nationale bien définie et cohérente et les quelques réussites isolées proviennent de l'imagination et de l'initiative d'individus. Au chapitre 3, on réfère à la désormais célèbre étude de Heather Menzies intitulée Women and the Chip: Case Studies of the Effects of Informatics on Employment in Canada (The Institute for Research on Public Policy, Montréal, 1981) qui constitue un cri d'alarme quant à l'avenir des travailleuses oeuvrant dans le secteur tertiaire (assurances, banques, etc.). Finalement, le lecteur pourra lire quelques mots sur les problèmes de santé reliés à l'emploi des écrans de visualisation (cathodiques). 
En résumé, ce livre est un résumé! Si le lecteur s'intéresse aux effets de la technologie sur les travailleurs et les emplois, s'il doit participer à des discussions en vue de l'élaboration de conventions collectives et qui impliquent la technologie, la santé et la sécurité au travail (pensons aux écrans cathodiques!), la formation et les habilités requises de la part des travailleurs oeuvrant en milieu automatisé, soit à l'usine, soit au bureau, alors ce document mérite d'être lu! Quant aux autres catégories de lecteurs, lisez ce livre si vous voulez rapidement et de façon concise - mais non de façon exhaustive - savoir ce qui se passe en matière de technologie nouvelle dans les pays suivants: le Japon, la Chine, la Malaisie, l'Australie, la NouvelleZélande, la Thaîlande, les Indes et les pays asiatiques en général, le Danemark, la Norvège, la Suède, la Finlande, la France, la Belgique, la Suisse, la République fédérale d'Allemagne, la Grande-Bretagne, l'Irlande, l'Italie, l'Espagne, l'Autriche, les pays de la Communauté européenne en général, la République démocratique allemande, la Tchécoslavaquie, la Roumanie, l'U.R.S.S. et l'Europe en général, le Brésil, l'Argentine, la Tanzanie, Israël, le Nigéria, les Etats-Unis et le Canada.

Rolland HURTUBISE

Ecole nationale d'administration publique

\section{PUBLICATIONS RÉCENTES RECENT PUBLICATIONS}

\section{Généralités}

«Dual Commitment and Labor Management Relationship Climates», by H.L. Angle and J.L. Perry, Academy of Management Journal, (Madison, Wisc.), vol. 29, no 1, 1986, pp. 31-50. «Ben Roberts: An Appreciation", by John Gennard, British Journal of Industrial Relations, (London, England), vol. XXIV, no 1, 1986, pp. 3-25.

«Italie: L'adaptation des relations professionnelles: les leçon de l'expérience des 15 dernières années", par G. Fajertag, Bulletin d'informations sociales, (Genève), vol. 3, no 4, 1985, pp. 401-405.

\section{Histoire du travail}

«Toward the History of the CIO. A Bibliographical Report», by R.H. Zieger, Labor History, (New York), vol. 26, no 4, 1985, pp. 485-516.

«Annual Bibliography on American Labor History 1984», by D. Swanson, Labor History, (New York), vol. 26, no 4, 1985, pp. 546-568.

«Recent Dissertations in American and European Labor History», by L. Cohen and H. Chapman, Labor History, (New York), vol. 26, no 4, 1985, pp. 569-576.

«Austromarxism on the International Scene», by Adolf Sturmthal, Reprint Series no 334, Institute of Labor and Industrial Relations, Univ. of Illinois at Urbana-Champaign, reprinted from «The Austrian Socialist Experiment Social Democracy and Austromarxism, 1918-1934». 